\title{
O UNIVERSO DISCENTE E O ENSINO DE BIOQUÍMICA.
}

\author{
Patrícia Fernanda Beckhauser ${ }^{1}$; Elzira Maria de Almeida e Ana Lúcia Bertarello Zeni²* \\ ${ }^{1}$ Acadêmica do curso de Ciências Biológicas \\ ${ }^{2}$ Professoras de Bioquímica do Departamento de Ciências Naturais, Centro de Ciências \\ Exatas e Naturais, Fundação Universidade Regional de Blumenau (FURB), Blumenau, \\ $\underline{\text { SC. }}$ \\ *anazeni@furb.br
}

\section{RESUMO}

Os diferentes cursos oferecidos na área de saúde na Universidade, recebem a cada semestre um grande número de alunos, originários de diferentes escolas de ensino médio e, cursos pré-vestibulares. A capacidade cognitiva e de abstração de cada aluno, bem como o grau de conhecimento prévio relativo à área, são fatores que geram diferenças individuais dentro de um mesmo grupo. Em algumas disciplinas do ciclo básico, que são comuns a vários cursos da área da saúde, e que estão inseridas no currículo nos primeiros semestres dos cursos de graduação, conhecimentos prévios são de fundamental importância para o desenvolvimento do conteúdo programático teórico e prático da disciplina. A Bioquímica é um exemplo de disciplina do ciclo básico que é oferecida em praticamente todos os cursos da área da Saúde e que requer principalmente noções de Química e Biologia celular. Entretanto, o conhecimento de um determinado grupo de alunos é bastante heterogêneo, o que exige do professor de Bioquímica, a criação de um ambiente que estimule a aplicação prática do que está sendo abordado no âmbito da disciplina. Este artigo discute os resultados de uma pesquisa realizada com alunos ingressantes na disciplina de Bioquímica de cursos de graduação da Fundação Universidade Regional de Blumenau-FURB, com o intuito de avaliar o grau de conhecimento prévio.

\section{ABSTRACT}

The different courses offered by the health area at University receive each semester a great number of students, from different high schools and pre-entry examination courses. The cognitive and abstraction capacity of each student, as well as the degree of relative previous knowledge to the area, are factors that generate individual differences inside a same group. In some disciplines of the basic cycle, which are common to several courses of the area of the health, and that are inserted in the curriculum in the first semesters of the graduation courses, previous knowledge are of fundamental importance for the development of the theoretical programmatic content and practice of the discipline. The Biochemistry is a discipline from the basic cycle that is offered in practically all of the health area courses, and requires mostly Chemistry notions and Cellular Biology. However, the knowledge sometimes acquired of a students certain group is a lot heterogeneous, what it demands of the Biochemistry teacher, the creation of an environment that stimulates the practice application than is being boarded in the scope of the discipline. This work argues the results of a research realized to the ingressants students in the Biochemistry discipline of the graduation courses of the Regional University Foundation of Blumenau - FURB, with the aim of evaluating the degree of previous knowledge. 


\section{INTRODUÇÃO}

Nos últimos anos os currículos de universidades que oferecem cursos na área da saúde vêm sofrendo a pressão da explosão do conhecimento científico e de seus meios de divulgação, o que resulta diretamente em uma sobrecarga do cognitivo e pulverização dos conhecimentos. A maioria dos currículos atuais é fragmentada em disciplinas isoladas, desvinculadas da realidade profissional, hipertrofiadas em conteúdos, voltadas para a formação tecnicista e para a especialidade, não formando profissionais que atendam as necessidades de saúde da sociedade [1]. Nesse processo de desvinculação entre as disciplinas básicas e profissionalizantes, fica comprometida de forma relevante a construção do conhecimento com o aluno, o que compromete a sua formação profissional.

O aumento do conhecimento nas diversas áreas das Ciências Biológicas, da Bioquímica e da Biologia Molecular, em particular, tem causado um dilema para os professores envolvidos com o ensino nestas áreas: enquanto o conhecimento aumenta, é impossível aumentar a carga horária das disciplinas proporcionalmente $[2,3]$. A disciplina de Bioquímica nos currículos tradicionais, apesar de ser apresentada com coerência e organização, normalmente é definida pelos estudantes como uma coleção de estruturas químicas e reações, dificilmente assimiladas e desintegradas de sua prática profissional [1].

$\mathrm{Na}$ área da saúde, as pesquisas no âmbito educacional são escassas. Estudos anteriores demonstraram que a avaliação educacional tem merecido menor atenção em periódicos destinados a profissionais da área da Saúde, que atuam como professores universitários. Existe uma menor produção científica sobre o tema e ausência de diagnósticos precisos a respeito das questões pedagógicas nos cursos de graduação da área da Saúde e, de modo especial à avaliação do rendimento acadêmico nos referidos cursos [4].

Com o objetivo de obter mais informações sobre os anseios e críticas dos alunos em relação à disciplina de Bioquímica, aplicamos um questionário com questões abrangendo aspectos que têm suscitado algumas observações quanto à dificuldade de entendimento, tanto pela disciplina em si, como pela falta de conhecimentos prévios, ou seja, obtidos no ensino médio ou cursinhos pré-vestibular, necessários para a aprendizagem.

\section{METODOLOGIA}

Aos cursos de Farmácia, Medicina e Ciências Biológicas da FURB, foi desenvolvido um questionário auto-aplicável com 9 questões abertas e fechadas (Anexo), nos semestres 2/2001 e 1/2002, totalizando uma amostragem de 171 alunos. O questionário individual foi disponibilizado pelo próprio professor da disciplina no primeiro dia de aula dos respectivos cursos, portanto antes de cursar a disciplina. O questionário era composto pelas mesmas questões independentemente do curso. A disciplina de Bioquímica é ministrada no $2^{\circ}$ semestre para o curso de Farmácia, no $1^{\circ}$ semestre para o curso de Medicina e no $5^{\circ}$ semestre para o curso de Ciências Biológicas. Os dados coletados através dos questionários foram organizados com o auxílio do programa Sphinx para análise e interpretação dos resultados.

\section{RESULTADOS E DISCUSSÃO}

Dos 171 alunos que responderam o questionário, 50 pertenciam ao curso de Medicina ( $1^{\mathrm{a}}$ fase), 54 ao curso de Biologia e 67 ao curso de Farmácia. Os estudantes eram provenientes de diversas escolas públicas e particulares, da cidade de Blumenau e também de outras cidades da região do Vale do Itajaí. Cerca de $30 \%$ dos alunos que responderam o questionário haviam freqüentado cursinho pré-vestibular por menos de 6 meses, 20\% não freqüentaram o cursinho, 35\% freqüentaram por mais de 6 meses e 15\% não responderam.

O gráfico da Fig. 1 mostra o número de alunos dos cursos participantes desta pesquisa. 0 maior número de alunos que respondeu o questionário é do curso de Biologia $(53,06 \%)$, $33,67 \%$ do curso de Farmácia e em menor número alunos do curso de Medicina $(12,24 \%)$. 


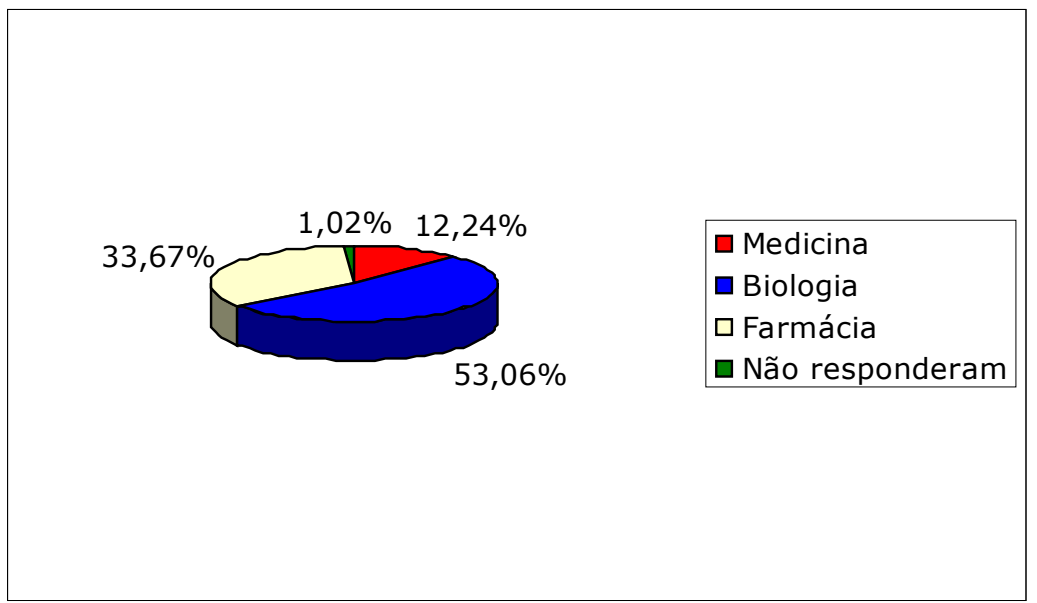

Figura 1. Relação dos cursos entrevistados.

A questão seguinte referia-se aos conteúdos que a Bioquímica abrange (Fig. 2). Aproximadamente $70 \%$ dos alunos que responderam o questionário não sabiam opinar sobre o que poderia ser estudado na disciplina, $27,55 \%$ tinham alguma noção de possíveis conteúdos, e somente $3,06 \%$ não responderam à questão.

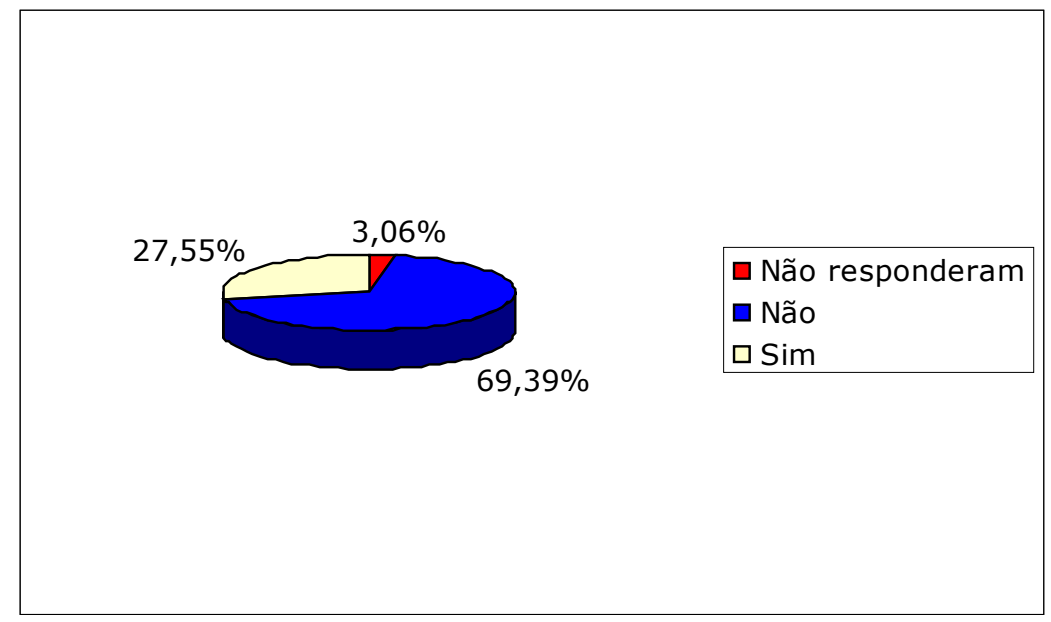

Figura 2. Você sabe quais conteúdos a Bioquímica abrange?

Dos entrevistados que tinham alguma noção dos conteúdos estudados pela Bioquímica (Fig. 2), 64,68\% responderam que seriam temas relacionados aos componentes químicos, organização e "transformações" da célula; 15,30\% responderam Biologia e Química; apenas $1,02 \%$ citaram conteúdos mais diversos, como Patologia e Ecologia, da mesma forma que a Biologia e a Física foram citados. $14,28 \%$ dos alunos não responderam à questão e somente 4,08\% não souberam como responder (Fig. 3).

Aos que tinham uma noção dos conteúdos que a disciplina abrange, a pergunta seguinte foi se os mesmos já haviam estudado anteriormente na vida escolar, algum dos conteúdos citados. $61 \%$ dos alunos disseram que não haviam estudado anteriormente os conteúdos, $24 \%$ disseram que sim e 15\% simplesmente não responderam (Fig. 4). Quanto ao período escolar em que teriam estudado os conteúdos, a maioria que cursou a disciplina no $1^{\circ}$ semestre da Faculdade (alunos do curso de Medicina, principalmente), atribui os ganhos de aprendizagem tanto à escola quanto ao cursinho enquanto que, os alunos que cursaram a disciplina a partir do $2^{\circ}$ semestre atribuem o grau de conhecimento à escola, ao cursinho e à universidade. Neste ponto é digno de nota o fato de que muitos alunos atribuem grande parte de seus conhecimentos ao cursinho (resultados não mostrados), o que evidencia que os cursinhos estão superando o ensino médio ao abordar alguns tópicos, que em alguns casos não são estudados no ensino médio ou não são abordados de forma tão significativa. 


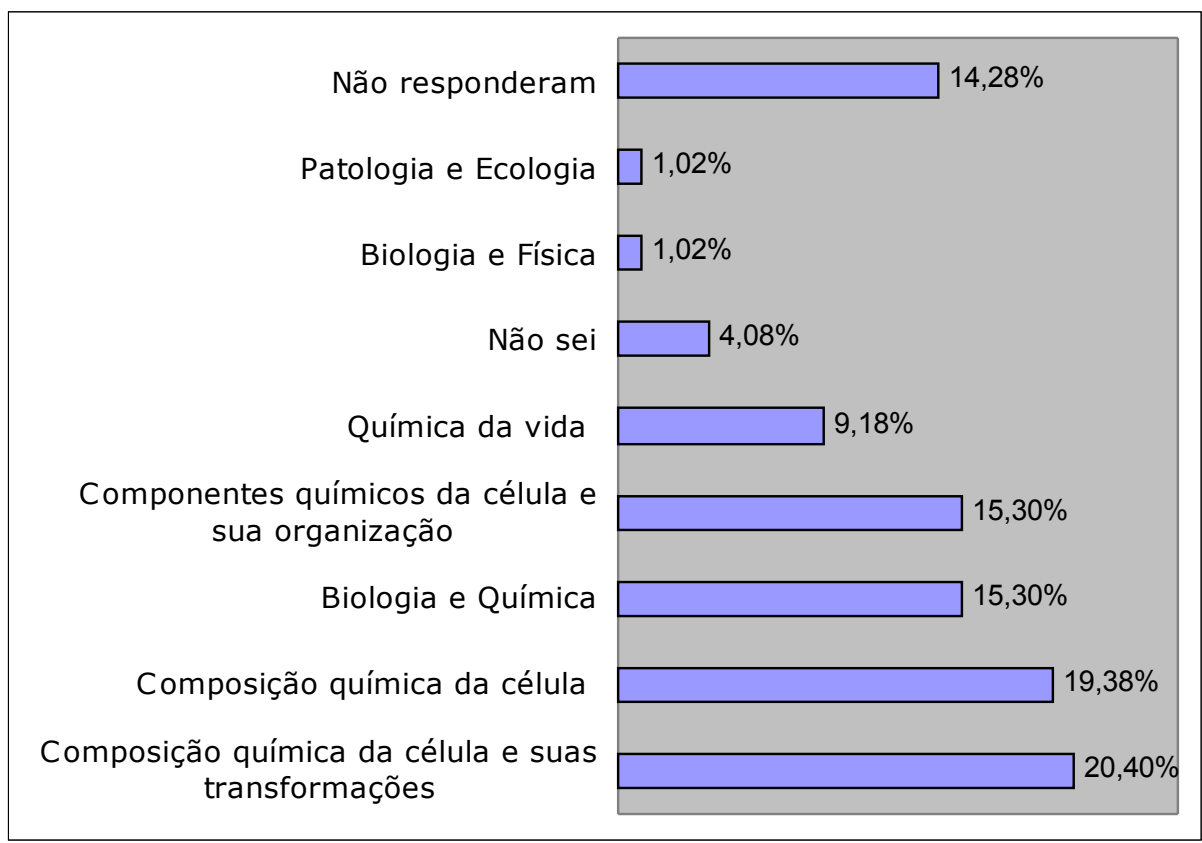

Figura 3. O que estuda a Bioquímica?

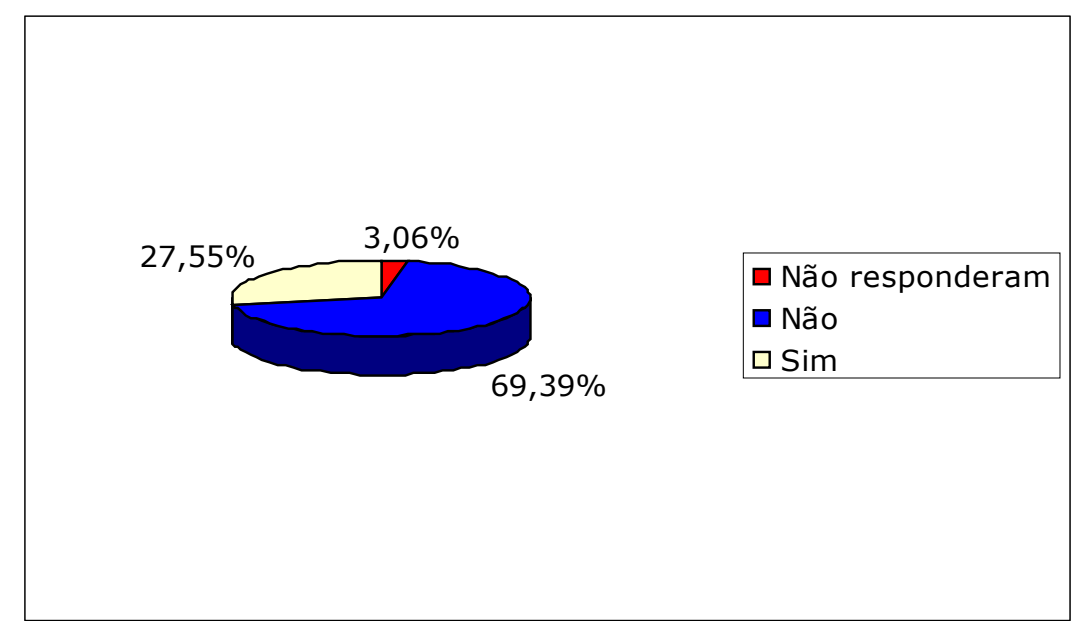

Figura 4. Estudou estes conteúdos antes?

O estudo da Bioquímica exige do aluno uma capacidade de abstração além de conhecimentos prévios de outras ciências para um bom desenvolvimento da disciplina. Considerando as características individuais dos alunos, o questionário continha também uma questão que sugeria vários assuntos para que fossem escolhidos quantos e quais o aluno dominava. A Fig. 5 mostra os resultados de uma lista destas habilidades e conteúdos. Embora as respostas tenham sido muito diversas, pôde-se observar que os alunos em sua grande maioria têm uma boa noção de Organização Celular (diferenciação entre procariotos e eucariotos) e Organelas celulares (respectivamente $13,78 \%$ e 13,57\%). As habilidades de Leitura e Interpretação de texto também foram citadas (12,32\% e $11,48 \%$ dos que responderam). Estas habilidades são de fundamental importância obviamente para o estudo de qualquer ciência e se faz ainda mais importante dentro de um curso de Bioquímica, onde é imprescindível a leitura para o acompanhamento da disciplina. $10,44 \%$ dos alunos que responderam, admitiram ter domínio sobre o reconhecimento de grupos funcionais. Certamente os alunos nesse momento do curso não têm noção de que a Química, Fisiologia e Biologia Celular são pré-requisitos naturais e agraciados durante o ensino médio, para o estudo da Bioquímica. Numa faixa que corresponde a $7-10 \%$ dos alunos que responderam o questionário citaram conteúdos relacionados à Química Geral, como pH, ionização, composição química celular e, como esperado, poucos alunos responderam dominar habilidades como esquematização de informações e leitura em inglês. 


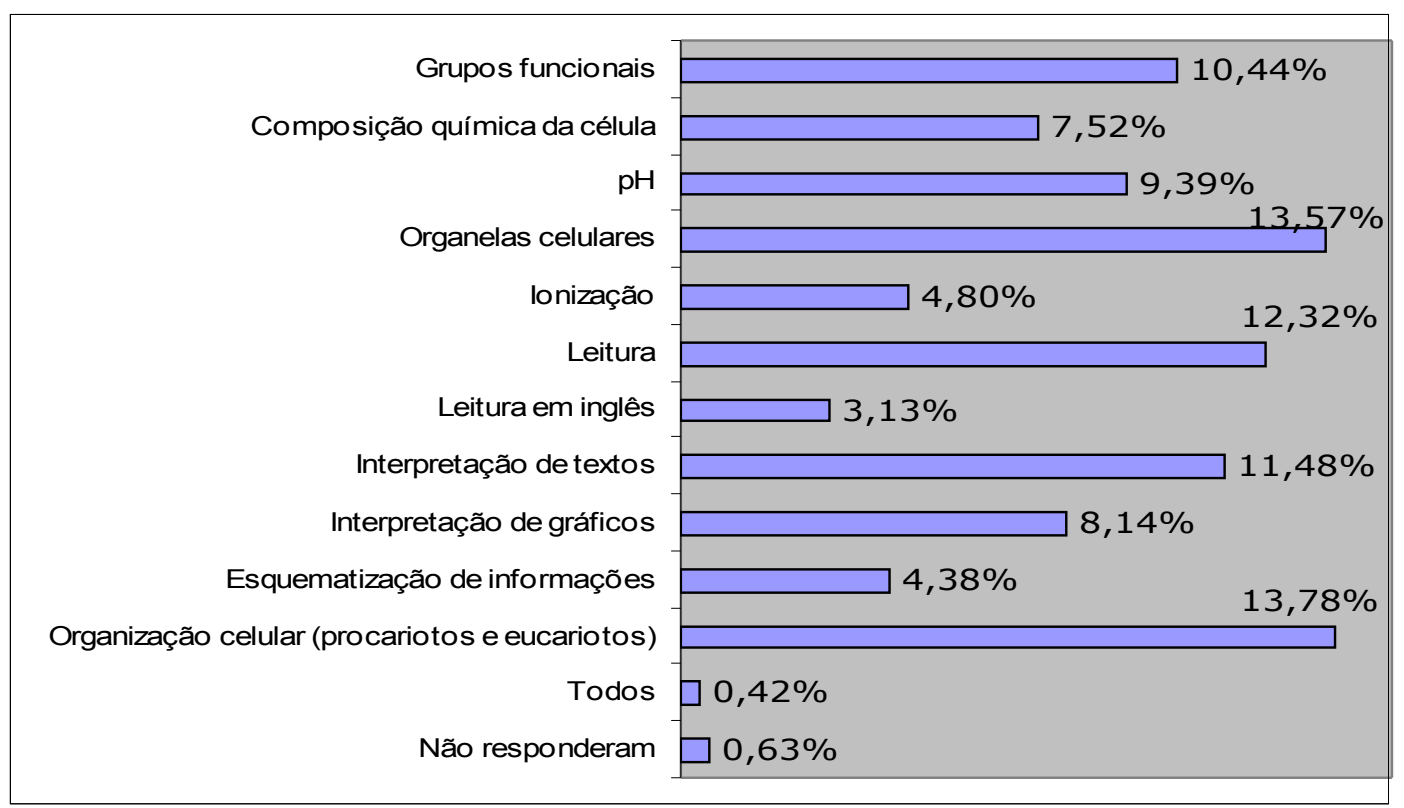

Figura 5. Dos assuntos e habilidades listados quais você domina?

O processo "ensinar e aprender" é um ciclo que envolve comportamentos intimamente relacionados, onde as ações de um provocam ou desencadeiam as reações do outro. Professor e aluno afetam-se mutuamente. Cada um desses elementos - professor e aluno - vem para sala de aula com uma bagagem própria, ou seja, cada um traz consigo uma história pessoal, valores, interesses, necessidades e dificuldades [5]. Conhecer alguns aspectos de como os alunos pensam torna-se extremamente importante para nortear todo o processo ensinoaprendizagem, baseado desta forma em conhecimento real do aluno que se encontra em sua sala de aula.

Quanto ao aspecto das relações entre as disciplinas dos currículos onde a Bioquímica está inserida, em sua grande maioria os alunos opinam que a Bioquímica se relaciona com outras disciplinas, principalmente, Química, Biologia Celular e Fisiologia. Em seguida foram citadas disciplinas como Histologia, Biofísica e Anatomia. Considerando que para o Curso de Ciências Biológicas a disciplina de Bioquímica é oferecida no $5^{\circ}$ semestre, disciplinas como Botânica, Zoologia, Genética e Biotecnologia, também foram relacionadas (resultados não mostrados).

Parte dos alunos tem dificuldade de correlacionar os conhecimentos aprendidos no início do curso, nas disciplinas do Ciclo Básico com o Ciclo Profissionalizante, divisão esta estabelecida pelos próprios currículos e não pelo conhecimento real. A integração horizontal é uma forma de distribuição das disciplinas nas escolas em geral, que fazem a segmentação dos processos como o meio para a elevação de sua eficiência. Essa segmentação gera alguns pontos negativos deste tipo de currículo. A experiência diária dos acadêmicos atesta que, quando se aproxima o momento de colocar a teoria em prática, aumentam as distâncias dos conhecimentos básicos necessários, levando as repetidas revisões de conteúdos já abordados, sendo os cursos caracterizados por muitos alunos, como repetitivos, gerando desinteresse. Esse desinteresse, reforçado pelo longo espaço temporal entre teoria e prática, é causa direta da desistência do curso por muitos alunos, que sequer tiveram a oportunidade de obter uma visão adequada da profissão que buscavam [6].

Portanto, iniciou-se uma série de discussões a esse respeito, onde foi realizada a seguinte pergunta: "Você acha a disciplina de Bioquímica importante devido à natureza de seu curso?". Em torno de $95 \%$ das respostas foram afirmativas, independente do curso, mostrando que cabe ao professor fazer a correlação entre a disciplina e sua aplicabilidade para cada curso, mostrando ao aluno a importância da Bioquímica no curso por eles escolhido. Em outras palavras os alunos sabem que a Bioquímica é importante, mas lhes falta segurança para explicar porquê. 


\section{CONSIDERAÇÕES FINAIS}

Mediante as respostas obtidas pelo questionário realizado, percebemos que, não existem diferenças importantes entre os cursos estudados e aparentemente, independentes do curso avaliado, existem dificuldades em responder com clareza qual é o objeto de estudo da Bioquímica. Quando os alunos conseguem responder esta questão, respondem na sua maioria como "a composição química", simplesmente. Sendo assim, quando questionados a respeito de já terem visto os conteúdos citados, respondem sobre a parte química, mais organelas celulares, porém ao se perguntar se dominam estes conteúdos, a maioria nega possuir este domínio.

Observa-se assim, que o universo discente é muito heterogêneo. Independente do curso escolhido, os alunos possuem alguma noção de assuntos que, do ponto de vista docente, a maioria deveria estar segura. Os alunos têm então intuitivamente a noção de que a Bioquímica é importante para o seu curso, talvez faltando somente que o professor tenha um maior contato com os seus alunos e descubra estas peculiaridades. Para aproximar os conteúdos teóricos da prática e o curso ora sendo realizado, com os alunos, deveríamos conhecer alguns aspectos de nossos alunos para enfim, não estarmos ensinando a nós mesmos e, sim aos alunos, conscientes de suas dificuldades e responsáveis também por sua própria aprendizagem.

\section{AGRADECIMENTOS}

Aos alunos dos cursos de Farmácia, Biologia e Medicina da FURB, espontaneamente participantes desta pesquisa, pela colaboração valiosa.

\section{REFERÊNCIAS BIBLIOGRÁFICAS}

[1] Vargas, L.H.M. (2001). A bioquímica e a aprendizagem baseada em problemas. Revista Brasileira de Ensino de Bioquímica e Biologia Molecular.

[2] Vieira, L. Q., Nicoli, J. R., Prado, V . F., Santoro, M. M., Teixeira, S. M. R., Bemquerer, M. \& Beirão S. L. (2001). Abordagem prática para o ensino de bioquímica. Revista Brasileira de Ensino de Bioquímica e Biologia Molecular.

[3] Regis, W.C.B.; Gonçalves, M.L.G.S.; Santoro, M. M. (2001). Avaliação das potencialidades de um curso prático de bioquímica na implementação do ensino teórico em cursos profissionalizantes Revista Brasileira de Ensino de Bioquímica e Biologia Molecular, Especial 1, 2001.

[4] Mezzaroba, L. (2000). Concepções de avaliação de professores e alunos de farmácia e bioquímica da Universidade Estadual de Londrina, Paraná. Revista Brasileira de Educação Médica. Rio de Janeiro. v. 24, $\mathrm{n}^{\circ}$ 3, out./dez.

[5] Moretto, R. \& Mansur, O. C. (2000). Ser professor: as múltiplas dimensões da docência. Revista Brasileira de Educação Médica. Rio de Janeiro. v.24, no 2, maio/set.

[6] Yasbeck, D. C. M., Azevedo, L. L., Siqueira, M. R. L. \& Menezes, V.M. (2000). Novos rumos para a educação médica. Revista Brasileira de Ensino em Educação Médica. Rio de Janeiro. v.24, no 2, maio/set. 


\section{Anexo}

\section{Questionário de sondagem}

Escola de origem:

Ano do término do ensino médio:

Tempo de cursinho:

1. O que estuda a Bioquímica?

2. A Bioquímica relaciona-se com outras disciplinas?

( ) $\operatorname{Sim}$ ( ) Não

Se responder sim, identifique-as:

3. Você acha a disciplina de Bioquímica importante devido a natureza de seu curso?

( ) Sim ( ) Não ( ) Muito pouco ( ) Não sabe

4. Você sabe quais conteúdos a Bioquímica abrange?

( ) $\operatorname{Sim}$ ( ) Não

Se responder sim, dê alguns exemplos?

5. Você estudou estes conteúdos?

( ) $\operatorname{Sim}$ ( ) Não

Se responder sim, onde?

6. Dos assuntos ou habilidades abaixo, quais delas você domina?
( ) grupos funcionais
( ) composição química da célula
( ) $\mathrm{pH}$
( ) organelas celulares
( ) ionização
( ) leitura
( ) leitura em inglês
( ) interpretação de textos
( ) interpretação de gráficos
( ) esquematização de informações
( ) organização celular (procariotos e eucariotos) 